\title{
An Improved Two-Step Method for Generalized Variational Inequalities
}

\author{
Haibin Chen \\ School of Management Science, Qufu Normal University, Rizhao, Shandong 276800, China \\ Correspondence should be addressed to Haibin Chen; chenhaibin508@163.com
}

Received 22 July 2013; Accepted 30 August 2013

Academic Editors: R. D. Chen and Y. Dai

Copyright (C) 2013 Haibin Chen. This is an open access article distributed under the Creative Commons Attribution License, which permits unrestricted use, distribution, and reproduction in any medium, provided the original work is properly cited.

\begin{abstract}
We propose an improved two-step extragradient algorithm for pseudomonotone generalized variational inequalities. It requires two projections at each iteration and allows one to take different stepsize rules. Moreover, from a geometric point of view, it is shown that the new method has a long stepsize, and it guarantees that the distance from the next iterative point to the solution set has a large decrease. Under mild conditions, we show that the method is globally convergent, and then the $R$-linearly convergent property of the method is proven if a projection-type error bound holds locally.
\end{abstract}

\section{Introduction}

Let $F$ be a multivalued mapping from $R^{n}$ into $2^{R^{n}}$ with nonempty values, where $R^{n}$ is a Euclidean space. Let $X$ be a nonempty, closed, and convex subset of the Euclidean space $R^{n}$. The generalized variational inequality, abbreviated as GVI, is to find a vector $x^{*} \in X$ such that there exists $\omega^{*} \in F\left(x^{*}\right)$ satisfying

$$
\left\langle\omega^{*}, x-x^{*}\right\rangle \geq 0, \quad \forall x \in X,
$$

where $\langle\cdot, \cdot\rangle$ stands for the inner product of vectors in $R^{n}$. The solution set of problem (1) is denoted by $X^{*}$. If the multivalued mapping $F$ is a single-valued mapping from $R^{n}$ to $R^{n}$, then the GVI collapses to the classical variational inequality problem [1-4].

For the problem GVI, we all know that it plays a significant role in economics and transportation equilibrium, engineering sciences, and so forth, and it has received considerable attention in the past decades [1,2,5-11]. Solution methods for GVI have been studied extensively. They can be roughly categorized into two popular approaches to attack the solution existence problem of the GVI. The first is analytic approaches. Instead of solving problem directly, the analytic approach reformulates the GVI as a well-studied mathematical problem first and then invokes an existence theorem for the latter problem [12]. The second is a constructive approach in which the existence can be verified by the behavior of the proposed method which will be considered in this paper.

To the best of our knowledge, the extragradient method $[2,13]$ is a popular constructive approach which was proposed by Korpelevich [13]. It has been proved that the method has a contract property; that is, the generated sequence $\left\{x^{k}\right\}$ by the method satisfies that

$$
\left\|x^{k+1}-x^{*}\right\| \leq\left\|x^{k}-x^{*}\right\|, \quad \forall k \geq 0,
$$

for any solution $x^{*}$ of the GVI. It should be noted that the proximal point algorithm also possesses this property [14].

In [15], the authors proposed a new type extragradient projection method for variational inequalities (VI). The method proposed in [15] required only two projections at each iteration and allowed one to take different stepsize rules. Moreover, it was shown that this method had a long stepsize, and it guaranteed that the distance from the next iterative point to the solution set had a large decrease. Some elementary numerical experiments showed its efficiency. Now a question is posed naturally: as the problem GVI is an extension of the problem VI, can this theory be extended to the GVI? This constitutes the main motivation of the paper.

In this paper, inspired by [15], we presented an improved extragradient method to the GVI problem. Under mild conditions, we first show that the generated sequence of the proposed method globally converges to the solution of 
the problem, and then we show that the method is $R$-linearly convergent if in addition a projection-type error bound holds locally. The rest of this paper is organized as follows. In Section 2, we give some related concepts and conclusions needed in the subsequent analysis. In Section 3, we present our designed algorithm and establish the convergence and convergent rate of the algorithm.

\section{Preliminaries}

In this section, we first give some related concepts and conclusions which are useful in the subsequent analysis. Let $x \in R^{n}$ and let $K$ be a nonempty closed convex set in $R^{n}$. A point $y_{0} \in K$ is said to be the orthogonal projection of $x$ onto $K$ if it is the closest point to $x$ in $K$; that is,

$$
y_{0}=\arg \min \{\|y-x\| \mid y \in K\},
$$

and denote $y_{0}$ by $P_{K}(x)$. The well-known properties of the projection operator are as follows.

Lemma 1 (see [16]). Let $K$ be a nonempty, closed, and convex subset in $R^{n}$. Then, for any $x, y \in R^{n}$ and $z \in K$, the following statements hold:

(i) $\left\langle P_{K}(x)-x, z-P_{K}(x)\right\rangle \geq 0$,

(ii) $\left\|P_{K}(x)-P_{K}(y)\right\|^{2} \leq\|x-y\|^{2}-\| P_{K}(x)-x+y-$ $P_{K}(y) \|^{2}$,

(iii) $\left\|P_{K}(x)-z\right\|^{2} \leq\|x-z\|^{2}-\left\|P_{K}(x)-x\right\|^{2}$,

(iv) $\left\langle z-x, z-P_{K}(x)\right\rangle \geq\left\|z-P_{K}(x)\right\|^{2}$.

Remark 2. In fact, (i) in Lemma 1 also provides a sufficient condition for a vector $u$ to be the projection of the vector $x$; that is, $u=P_{K}(x)$ if and only if

$$
\langle u-x, z-u\rangle \geq 0, \quad \forall z \in K .
$$

Lemma 3. Let $K$ be a nonempty, closed, and convex subset in $R^{n}$. For any $x, d \in R^{n}$ and $\alpha \geq 0$, define

$$
x(\alpha)=P_{K}(x-\alpha d) .
$$

Then, $\langle d, x-x(\alpha)\rangle$ is nondecreasing for $\alpha>0$.

Lemma 4. Let $K$ be a nonempty closed and convex subset in $R^{n}$. For any $x, d \in R^{n}$ and $\alpha \geq 0$, define

$$
\varphi(\alpha)=\min \left\{\|y-x+\alpha d\|^{2} \mid y \in K\right\} .
$$

Then,

$$
\varphi^{\prime}(\alpha)=2\langle d, x(\alpha)-x+\alpha d\rangle .
$$

Definition 5. Let $K$ be a nonempty subset of $R^{n}$. The multivalued mapping $F: K \rightarrow 2^{R^{n}}$ is said to be

(i) monotone if and only if

$$
\langle u-v, x-y\rangle \geq 0, \quad \forall x, y \in K, u \in F(x), v \in F(y),
$$

(ii) pseudomonotone if and only if, for any $x, y \in K, u \in$ $F(x), v \in F(y)$,

$$
\langle u, y-x\rangle \geq 0 \Longrightarrow\langle v, y-x\rangle \geq 0
$$

To proceed, we need the following definition for a multivalued mapping $F$.

Definition 6. Let $K$ be a nonempty, closed, and convex subset of $R^{n}$. A multivalued mapping $F: K \rightarrow 2^{R^{n}}$ is said to be

(i) upper semicontinuous at $x \in K$ if, for every open set $V$ containing $F(x)$, there is an open set $U$ containing $x$ such that $F(y) \subset V$ for all $y \in K \cap U$;

(ii) lower semicontinuous at $x \in K$ if, given any sequence $x^{k}$ converging to $x$ and any $y \in F(x)$, there exists a sequence $y^{k} \in F\left(x^{k}\right)$ that converges to $y$;

(iii) continuous at $x \in K$ if it is both upper semicontinuous and lower semicontinuous at $x$.

To end this section, we state the assumptions needed in the subsequent analysis.

Assumption 7. Let $X$ be a nonempty, closed, and convex subset of $R^{n}$. And we assume

(i) $X^{*}$ is nonempty;

(ii) the multivalued mapping $F: X \rightarrow 2^{R^{n}}$ is pseudomonotone and continuous on $X$ with compact convex values.

\section{Main Results}

For any $x \in R^{n}$ and $\xi \in F(x)$, set

$$
r(x, \xi)=x-P_{X}(x-\xi)
$$

Then the projection residue $r(x, \xi)$ can verify the solution set of the GVI [17].

Proposition 8. Let $x \in X$ and $\xi \in F(x)$. Then $(x, \xi)$ solves the problem (1) if and only if

$$
x=P_{X}(x-\xi) .
$$

The basic idea of the designed algorithm is as follows. At each step of the algorithm, compute the projection residue $r\left(x^{k}, \xi^{k}\right)$ at iterate $x^{k}$. If $r\left(x^{k}, \xi^{k}\right)=0$, then stop with $x^{k}$ being a solution of the GVI; otherwise, find a trial point $y^{k}$ by a back-tracking search at $x^{k}$ along the residue $r\left(x^{k}, \xi^{k}\right)$, and the new iterate is obtained by using a projection. Repeat this process until the projection residue is a zero vector.

Now, we describe carefully our algorithmic framework for solving GVI. 
Algorithm 9. Choose $\sigma, \gamma \in(0,1), x^{0} \in X, k=0$.

Step 1. Given the current iterate $x^{k}$, if $\left\|r\left(x^{k}, \xi^{k}\right)\right\|=0$ for some $\xi^{k} \in F\left(x^{k}\right)$, stop; else take any $\xi^{k} \in F\left(x^{k}\right)$ and compute

$$
z^{k}=P_{X}\left(x^{k}-\xi^{k}\right) \text {. }
$$

Let

$$
y^{k}=\left(1-\eta_{k}\right) x^{k}+\eta_{k} z^{k},
$$

where $\eta_{k}=\gamma^{m_{k}}$, with $m_{k}$ being the smallest nonnegative integer $m$ satisfying: $\exists \zeta^{k} \in F\left(x^{k}-\gamma^{m} r\left(x^{k}, \xi^{k}\right)\right)$ such that

$$
\left\langle\zeta^{k}, r\left(x^{k}, \xi^{k}\right)\right\rangle \geq \sigma\left\|r\left(x^{k}, \xi^{k}\right)\right\|^{2} .
$$

Step 2. Let $x^{k+1}=P_{X}\left(x^{k}-\alpha_{k} \zeta^{k}\right)$, where $\zeta^{k}$ is chosen as in (14) and $\alpha_{k}$ is chosen such that

$$
\begin{gathered}
\alpha_{k} \geq \frac{\left\langle\zeta^{k}, x^{k}-y^{k}\right\rangle}{\left\|\zeta^{k}\right\|^{2}}, \\
\left\langle\zeta^{k}, P_{X}\left(x^{k}-\alpha_{k} \zeta^{k}\right)-y^{k}\right\rangle \geq 0 .
\end{gathered}
$$

Step 3. Set $k=k+1$ and go to Step 1 .

First, we give a conclusion which addresses the feasibility of the stepsize rule (14), that is, the existence of point $\zeta^{k}$.

Lemma 10. If $x^{k}$ is not a solution of the problem (1), then there exists the smallest nonnegative integer $m$ satisfying (14) under Assumption 7.

Proof. By the definition of $r\left(x^{k}, \xi^{k}\right)$ and Lemma 1, it follows that

$$
\left\langle P_{X}\left(x^{k}-\xi^{k}\right)-\left(x^{k}-\xi^{k}\right), x^{k}-P_{X}\left(x^{k}-\xi^{k}\right)\right\rangle \geq 0,
$$

which implies

$$
\left\langle\xi^{k}, r\left(x^{k}, \xi^{k}\right)\right\rangle \geq\left\|r\left(x^{k}, \xi^{k}\right)\right\|^{2}>0 .
$$

Since $\gamma \in(0,1)$, we get

$$
\lim _{m \rightarrow \infty}\left(x^{k}-\gamma^{m} r\left(x^{k}, \xi^{k}\right)\right)=x^{k} .
$$

Combining this with the fact that $F$ is lower semicontinuous, we know that there exists $\zeta^{m} \in F\left(x^{k}-\gamma^{m} r\left(x^{k}, \xi^{k}\right)\right)$ such that

$$
\lim _{m \rightarrow \infty} \zeta^{m}=\xi^{k}
$$

Hence, by (18), one has

$$
\begin{aligned}
\lim _{m \rightarrow \infty}\left\langle\zeta^{m}, r\left(x^{k}, \xi^{k}\right)\right\rangle & =\left\langle\xi^{k}, r\left(x^{k}, \xi^{k}\right)\right\rangle \\
& \geq\left\|r\left(x^{k}, \xi^{k}\right)\right\|^{2}>0 .
\end{aligned}
$$

This completes the proof.
Now, for the sake of convenience, we define

$$
x^{k}(\alpha)=P_{X}\left(x^{k}-\alpha \zeta^{k}\right),
$$

$$
\varphi_{k}(\alpha)=2 \alpha\left\langle\zeta^{k}, x^{k}-y^{k}\right\rangle+\left\|x^{k}-\alpha \zeta^{k}-x^{k}(\alpha)\right\|^{2}-\alpha^{2}\left\|\zeta^{k}\right\|^{2}
$$

for $\alpha \geq 0$. Then, we have the following result.

Lemma 11. For the generated sequence $\left\{x^{k}\right\}$ in Algorithm 9, it holds that

$$
\left\|x^{k+1}-x^{*}\right\|^{2} \leq\left\|x^{k}-x^{*}\right\|^{2}-\varphi_{k}\left(\alpha_{k}\right)
$$

under Assumption 7, where $x^{*}$ is a point in $X^{*}$.

Proof. By Lemma 1 (iii) and the iterative process of Algorithm 9, we have

$$
\begin{gathered}
\left\|x^{k+1}-x^{*}\right\|^{2} \leq\left\|x^{k}-x^{*}-\alpha_{k} \zeta^{k}\right\|^{2}-\left\|x^{k}-\alpha_{k} \zeta^{k}-x^{k+1}\right\|^{2} \\
\leq\left\|x^{k}-x^{*}\right\|^{2}+\alpha_{k}^{2}\left\|\zeta^{k}\right\|^{2}-2 \alpha_{k}\left\langle\zeta^{k}, x^{k}-x^{*}\right\rangle \\
-\left\|x^{k}-\alpha_{k} \zeta^{k}-x^{k+1}\right\|^{2} .
\end{gathered}
$$

Since $x^{*} \in X^{*}$, it follows that there exists $\omega^{*} \in F\left(x^{*}\right)$ such that

$$
\left\langle\omega^{*}, y^{k}-x^{*}\right\rangle \geq 0 .
$$

Combining this and the fact that $F$ is pseudomonotone, one has

$$
\left\langle\zeta^{k}, y^{k}-x^{*}\right\rangle \geq 0 .
$$

On the other hand,

$$
\left\langle\zeta^{k}, x^{k}-x^{*}\right\rangle=\left\langle\zeta^{k}, x^{k}-y^{k}\right\rangle+\left\langle\zeta^{k}, y^{k}-x^{*}\right\rangle .
$$

By (27), we have

$$
-2 \alpha_{k}\left\langle\zeta^{k}, x^{k}-x^{*}\right\rangle \leq-2 \alpha_{k}\left\langle\zeta^{k}, x^{k}-y^{k}\right\rangle .
$$

It is obvious that

$$
\begin{aligned}
\left\|x^{k+1}-x^{*}\right\|^{2} \leq & \left\|x^{k}-x^{*}-\alpha_{k} \zeta^{k}\right\|^{2}-\left\|x^{k}-\alpha_{k} \zeta^{k}-x^{k+1}\right\|^{2} \\
\leq & \left\|x^{k}-x^{*}\right\|^{2}+\alpha_{k}^{2}\left\|\zeta^{k}\right\|^{2}-2 \alpha_{k}\left\langle\zeta^{k}, x^{k}-x^{*}\right\rangle \\
& -\left\|x^{k}-\alpha_{k} \zeta^{k}-x^{k+1}\right\|^{2} \\
\leq & \left\|x^{k}-x^{*}\right\|^{2}+\alpha_{k}^{2}\left\|\zeta^{k}\right\|^{2}-2 \alpha_{k}\left\langle\zeta^{k}, x^{k}-y^{k}\right\rangle \\
& -\left\|x^{k}-\alpha_{k} \zeta^{k}-x^{k+1}\right\|^{2} .
\end{aligned}
$$

So, by the definition of $\varphi_{k}$, we obtain

$$
\left\|x^{k+1}-x^{*}\right\|^{2} \leq\left\|x^{k}-x^{*}\right\|^{2}-\varphi_{k}\left(\alpha_{k}\right),
$$

and the proof is completed. 
To prove the existence of $\alpha_{k}$ in Step 2 of Algorithm 9, we first consider the following optimization problem:

$$
\max \left\{\varphi_{k}(\alpha) \mid \alpha \geq 0\right\},
$$

which is very necessary for the feasibility proof of $\alpha_{k}$. By Lemma 4 and the definition of $\varphi_{k}(\alpha)$, it follows that

$$
\begin{aligned}
\varphi_{k}^{\prime}(\alpha) & =2\left\langle\zeta^{k}, x^{k}-y^{k}\right\rangle+2\left\langle\zeta^{k}, x^{k}(\alpha)-x^{k}+\alpha \zeta^{k}\right\rangle-2 \alpha\left\|\zeta^{k}\right\|^{2} \\
& =2\left\langle\zeta^{k}, x^{k}-y^{k}\right\rangle+2\left\langle\zeta^{k}, x^{k}(\alpha)-x^{k}\right\rangle \\
& =2\left\langle\zeta^{k}, P_{X}\left(x^{k}-\alpha \zeta^{k}\right)-y^{k}\right\rangle .
\end{aligned}
$$

Note that $\varphi_{k}(0)=0$ and

$$
\begin{aligned}
\varphi_{k}^{\prime}(0) & =2\left\langle\zeta^{k}, x^{k}-y^{k}\right\rangle=2 \eta_{k}\left\langle\zeta^{k}, r\left(x^{k}, \xi^{k}\right)\right\rangle \\
& \geq 2 \sigma \eta_{k}\left\|r\left(x^{k}, \xi^{k}\right)\right\|^{2}>0,
\end{aligned}
$$

where the first inequality follows from (14). Then

$$
\max \left\{\varphi_{k}(\alpha) \mid \alpha \geq 0\right\}>0
$$

if the maximal value exists. By Lemma 3 , we know that $\varphi_{k}^{\prime}(\alpha)$ is nonincreasing and continuous for $\alpha \geq 0$. So if $\varphi_{k}^{\prime}(\alpha)=0$ is solvable on $(0,+\infty)$, then its solution coincides with the solution to the optimization problem

$$
\max \left\{\varphi_{k}(\alpha) \mid \alpha \geq 0\right\} .
$$

Next, from a geometric point of view, we will show that the equation $\varphi_{k}^{\prime}(\alpha)=0$ is solvable on $(0,+\infty)$.

Lemma 12. If $x^{k}$ is not a solution of the problem (1), then equation $\varphi_{k}^{\prime}(\alpha)=0$ is solvable for $\alpha \geq 0$ under the Assumption 7.

Proof. For the sake of simplicity, we first define halfspaces as follows:

$$
\begin{aligned}
& H_{k}^{1}=\left\{x \in R^{n} \mid\left\langle\zeta^{k}, x-y^{k}\right\rangle<0\right\}, \\
& H_{k}^{2}=\left\{x \in R^{n} \mid\left\langle\zeta^{k}, x-y^{k}\right\rangle>0\right\}, \\
& H_{k}^{3}=\left\{x \in R^{n} \mid\left\langle\zeta^{k}, x-y^{k}\right\rangle=0\right\},
\end{aligned}
$$

where $\zeta^{k}$ is the same as in (14).

Since $x^{k} \notin X^{*}$, by the iterative process of Algorithm 9, one has

$$
\begin{aligned}
\left\langle\zeta^{k}, z^{k}-y^{k}\right\rangle & =\left(1-\eta_{k}\right)\left\langle\zeta^{k}, z^{k}-x^{k}\right\rangle \\
& \leq-\left(1-\eta_{k}\right) \sigma\left\|r\left(x^{k}, \xi^{k}\right)\right\|^{2}<0,
\end{aligned}
$$

and since

$$
\left\langle\zeta^{k}, x^{k}-y^{k}\right\rangle=\eta_{k}\left\langle\zeta^{k}, r\left(x^{k}, \xi^{k}\right)\right\rangle \geq \eta_{k} \sigma\left\|r\left(x^{k}, \xi^{k}\right)\right\|^{2}>0,
$$

we know that $H_{k}^{1} \cap X, H_{k}^{2} \cap X$, and $H_{k}^{3} \cap X$ are all nonempty convex sets, respectively. Let

$$
\alpha_{k}^{1}=\frac{\left\langle\zeta^{k}, x^{k}-y^{k}\right\rangle}{\left\|\zeta^{k}\right\|^{2}} .
$$

It is obvious that

$$
\left\langle\zeta^{k}, x^{k}-\alpha_{k}^{1} \zeta^{k}-y^{k}\right\rangle=0 .
$$

By the fact that $\left\langle\zeta^{k}, x^{k}-\alpha \zeta^{k}-y^{k}\right\rangle$ is nonincreasing for $\alpha>0$, we have

$$
\left\langle\zeta^{k}, x^{k}-\alpha \zeta^{k}-y^{k}\right\rangle<0
$$

for $\alpha>\alpha_{k}^{1}$.

Now, let $Q$ be any point in $H_{k}^{1} \cap X$ and let $P$ be any point in $H_{k}^{2} \cap X$. In the triangle composed by the points $Q, P$, and $O(\alpha)=x^{k}-\alpha \zeta^{k}$, we denote the inner corners at points $Q$ and $P$ by $\beta_{Q}$ and $\beta_{P}$, respectively. By geometric consideration, if $\alpha>\alpha_{k}^{1}$ is sufficiently large, we obtain

$$
\beta_{P}<\beta_{\mathrm{Q}}, \quad\|O(\alpha) Q\|<\|O(\alpha) P\| .
$$

By the arbitrariness of $P \in H_{k}^{2} \cap X$ and the definition of projection, there exists $\alpha_{k}^{\prime}>\alpha_{k}^{1}$ satisfying

$$
P_{X}\left(x^{k}-\alpha_{k}^{\prime} \zeta^{k}\right) \in H_{k}^{1} .
$$

On the other hand, by (39), it follows that

$$
\left\langle\zeta^{k}, P_{X}\left(x^{k}-0 \zeta^{k}\right)-y^{k}\right\rangle=\left\langle\zeta^{k}, x^{k}-y^{k}\right\rangle>0,
$$

which implies that $P_{X}\left(x^{k}-0 \zeta^{k}\right) \in H_{k}^{2}$. Then, by the continuity of the projection operator, there exists $\alpha_{k}^{2} \in\left(0, \alpha_{k}^{\prime}\right)$ such that

$$
x^{k}\left(\alpha_{k}^{2}\right)=P_{X}\left(x^{k}-\alpha_{k}^{2} \zeta^{k}\right) \in H_{k}^{3} \cap X,
$$

which means that

$$
\left\langle\zeta^{k}, P_{X}\left(x^{k}-\alpha_{k}^{2} \zeta^{k}\right)-y^{k}\right\rangle=0 .
$$

So $\varphi_{k}^{\prime}\left(\alpha_{k}^{2}\right)=0$, and the desired result follows.

In order to maintain consistency in the sequel, we denote the smallest positive solution to the equation $\varphi_{k}^{\prime}(\alpha)=0$ by $\alpha_{k}^{2}$. Then, $\alpha_{k}^{2}$ is the smallest positive solution to

$$
\max \left\{\varphi_{k}(\alpha) \mid \alpha \geq 0\right\} .
$$

Lemma 13. Take $\alpha_{k}=\alpha_{k}^{2}$ in Algorithm 9; then $\alpha_{k}$ satisfies (15) and (16) under Assumption 7.

Proof. By the proof of Lemma 12, it is obvious that (16) holds. On the other hand, since $\varphi_{k}^{\prime}\left(\alpha_{k}^{2}\right)=0$ and

$$
\varphi_{k}^{\prime}(\alpha)=2\left\langle\zeta^{k}, x^{k}-y^{k}\right\rangle+2\left\langle\zeta^{k}, x^{k}(\alpha)-x^{k}\right\rangle,
$$


one has

$$
\left\langle\zeta^{k}, x^{k}-y^{k}\right\rangle=\left\langle\zeta^{k}, x^{k}-x^{k}\left(\alpha_{k}^{2}\right)\right\rangle>0,
$$

where the last inequality follows from (39). By the fact that the projection operator is nonexpansive, we have

$$
\begin{aligned}
\left\langle\zeta^{k}, x^{k}-y^{k}\right\rangle & =\left\langle\zeta^{k}, x^{k}-x^{k}\left(\alpha_{k}^{2}\right)\right\rangle \\
& =\left\langle\zeta^{k}, x^{k}-P_{X}\left(x^{k}-\alpha_{k}^{2} \zeta^{k}\right)\right\rangle \\
& \leq\left\|\zeta^{k}\right\|\left\|x^{k}-P_{X}\left(x^{k}-\alpha_{k}^{2} \zeta^{k}\right)\right\| \\
& \leq \alpha_{k}^{2}\left\|\zeta^{k}\right\|^{2},
\end{aligned}
$$

which implies

$$
\alpha_{k}^{2} \geq \frac{\left\langle\zeta^{k}, x^{k}-y^{k}\right\rangle}{\left\|\zeta^{k}\right\|^{2}}=\alpha_{k}^{1}
$$

and (15) holds. The desired result follows.

Since $\varphi_{k}^{\prime}(\alpha)>0$ for all $\alpha \in\left[0, \alpha_{k}^{2}\right)$, we know that (15) and (16) hold for any $\alpha_{k} \in\left[\alpha_{k}^{1}, \alpha_{k}^{2}\right]$. That is to say, we can take $\alpha_{k} \in\left[\alpha_{k}^{1}, \alpha_{k}^{2}\right]$ in Algorithm 9, which shows the feasibility and flexibility of the method. Of course, by Lemma 11, we know that $\alpha_{k}=\alpha_{k}^{2}$ in Algorithm 9 is a better stepsize in the sense that the distance between the next iterate point and $X^{*}$ has a large decrease at each iteration, which shows theoretically the superiority of the method.

Theorem 14. Suppose Assumption 7 holds. If Algorithm 9 generates an infinite sequence $\left\{x^{k}\right\}$, then it converges to a solution of GVI and

$$
\lim _{k \rightarrow \infty}\left\|r\left(x^{k}, \xi^{k}\right)\right\|=0 .
$$

Proof. For each iterative process, by the stepsize rule, we have

$$
\varphi_{k}\left(\alpha_{k}\right) \geq \varphi_{k}\left(\alpha_{k}^{1}\right) \text {. }
$$

Combining this and Lemma 11 one has

$$
\begin{aligned}
\left\|x^{k+1}-x^{*}\right\|^{2} \leq & \left\|x^{k}-x^{*}\right\|^{2}-\varphi_{k}\left(\alpha_{k}\right) \\
\leq & \left\|x^{k}-x^{*}\right\|^{2}-\varphi_{k}\left(\alpha_{k}^{1}\right) \\
= & \left\|x^{k}-x^{*}\right\|^{2}+\left(\alpha_{k}^{1}\right)^{2}\left\|\zeta^{k}\right\|^{2}-2 \alpha_{k}^{1}\left\langle\zeta^{k}, x^{k}-y^{k}\right\rangle \\
& -\left\|x^{k}-x^{k}\left(\alpha_{k}^{1}\right)-\alpha_{k}^{1} \zeta^{k}\right\|^{2}=\left\|x^{k}-x^{*}\right\|^{2} \\
& +\frac{\left\langle\zeta^{k}, x^{k}-y^{k}\right\rangle^{2}}{\left\|\zeta^{k}\right\|^{2}}-2 \frac{\left\langle\zeta^{k}, x^{k}-y^{k}\right\rangle^{2}}{\left\|\zeta^{k}\right\|^{2}} \\
& -\left\|x^{k}-x^{k}\left(\alpha_{k}^{1}\right)-\alpha_{k}^{1} \zeta^{k}\right\|^{2}=\left\|x^{k}-x^{*}\right\|^{2} \\
& -\left(\alpha_{k}^{1}\right)^{2}\left\|\zeta^{k}\right\|^{2}-\left\|x^{k}-x^{k}\left(\alpha_{k}^{1}\right)-\alpha_{k}^{1} \zeta^{k}\right\|^{2} \\
\leq & \left\|x^{k}-x^{*}\right\|^{2}-\left(\alpha_{k}^{1}\right)^{2}\left\|\zeta^{k}\right\|^{2},
\end{aligned}
$$

where $x^{*}$ is chosen from $X^{*}$. Hence, the sequence $\left\{\left\|x^{k}-x^{*}\right\|\right\}$ is nonincreasing and $\left\{\left\|x^{k}-x^{*}\right\|\right\}$ is bounded. Then, it follows that

$$
\lim _{k \rightarrow \infty}\left(\alpha_{k}^{1}\left\|\zeta^{k}\right\|\right)=0
$$

from which we obtain

$$
\lim _{k \rightarrow \infty} \frac{\left\langle\zeta^{k}, x^{k}-y^{k}\right\rangle}{\left\|\zeta^{k}\right\|}=0 .
$$

Since $F$ is continuous with compact values, Proposition 3.11 in [18] implies that $\left\{F\left(y^{k}\right): k \in N\right\}$ is a bounded set, and so the sequence $\left\{\zeta^{k}: \zeta^{k} \in F\left(y^{k}\right)\right\}$ is bounded. Hence

$$
\lim _{k \rightarrow \infty}\left\langle\zeta^{k}, x^{k}-y^{k}\right\rangle=0
$$

By the iterative process of Algorithm 9 and since

$$
\left\langle\zeta^{k}, x^{k}-y^{k}\right\rangle \geq \eta_{k} \sigma\left\|r\left(x^{k}, \xi^{k}\right)\right\|^{2},
$$

we have

$$
\lim _{k \rightarrow \infty}\left(\eta_{k} \sigma\left\|r\left(x^{k}, \xi^{k}\right)\right\|^{2}\right)=0 .
$$

Without loss of generality, if $\lim _{k \rightarrow \infty} \eta_{k} \neq 0$, by (60), one has

$$
\lim _{k \rightarrow \infty} r\left(x^{k}, \xi^{k}\right)=0
$$

and the desired result can be obtained.

On the other hand, suppose $\lim _{k \rightarrow \infty} \eta_{k}=0$. By the fact that $\left\{\left\|x^{k}\right\|\right\}$ is bounded, so it has a convergent subsequence $x^{k_{j}}$, and the limit is denoted by $\bar{x}$; that is,

$$
\lim _{j \rightarrow \infty} x^{k_{j}}=\bar{x}
$$

Therefore,

$$
\begin{aligned}
y^{k_{j}} & =\left(1-\eta_{k_{j}}\right) x^{k_{j}}+\eta_{k_{j}} z^{k_{j}} \\
& =x^{k_{j}}-\eta_{k_{j}} r\left(x^{k_{j}}, \xi^{k_{j}}\right) \longrightarrow \bar{x}, \text { as } j \longrightarrow \infty .
\end{aligned}
$$

Since $F$ is continuous on $X$, so for all $\xi \in F(\bar{x})$ we know that there exist $\xi^{k_{j}} \in F\left(x^{k_{j}}\right)$ such that

$$
\lim _{j \rightarrow \infty} \xi^{k_{j}}=\xi
$$

and there exist $\zeta^{k_{j}} \in F\left(x^{k_{j}}-\left(\eta_{k_{j}} / \gamma\right) r\left(x^{k_{j}}, \xi^{k_{j}}\right)\right)$ such that

$$
\lim _{j \rightarrow \infty} \zeta^{k_{j}}=\xi
$$

Observing the definition of $\eta_{k_{j}}$ and $\zeta^{k_{j}} \in F\left(x^{k_{j}}-\left(\eta_{k_{j}} /\right.\right.$ ү) $\left.r\left(x^{k_{j}}, \xi^{k_{j}}\right)\right)$, it implies that

$$
\left\langle\zeta^{k_{j}}, r\left(x^{k_{j}}, \xi^{k_{j}}\right)\right\rangle<\sigma\left\|r\left(x^{k_{j}}, \xi^{k_{j}}\right)\right\|^{2} .
$$


Let $j \rightarrow \infty$ in (66), and we obtain

$$
\langle\xi, r(\bar{x}, \xi)\rangle<\sigma\|r(\bar{x}, \xi)\|^{2}
$$

By Lemma 1 (iv), it follows that

$$
\begin{aligned}
\langle\xi, r(\bar{x}, \xi)\rangle & =\left\langle\bar{x}-(\bar{x}-\xi), \bar{x}-P_{X}(\bar{x}-\xi)\right\rangle \\
& \geq\left\|\bar{x}-P_{X}(\bar{x}-\xi)\right\|^{2}=\|r(\bar{x}, \xi)\|^{2} .
\end{aligned}
$$

Combining this with (67) we have

$$
r(\bar{x}, \xi)=0
$$

which implies that $\bar{x} \in X^{*}$. By the fact that $\left\{\left\|x^{k_{j}}-\bar{x}\right\|\right\}$ converges to zero and the whole sequence $\left\{\left\|x^{k}-\bar{x}\right\|\right\}$ is nonincreasing, we obtain that $\lim _{k \rightarrow \infty}\left\|x^{k}-\bar{x}\right\|=0$; that is, $\lim _{k \rightarrow \infty} x^{k}=\bar{x}$. And the desired result holds.

The study of the following results is in the spirit of convergence rate results in $[19,20]$ in $R^{n}$, which are based on error bounds. The research on error bounds is a large topic in mathematical programming. One can refer to the surveys [21] for some sufficient conditions ensuring the existence of error bounds and for the roles played by error bounds in the convergence analysis of iterative algorithms.

Now, we first give the definition of Lipschitz continuous for a multivalued mapping.

Definition 15. A multivalued mapping $F: X \rightarrow 2^{R^{n}}$ is said to be Lipschitz continuous if there exists a constant $L>0$ such that

$$
H(F(x), F(y)) \leq L\|x-y\|, \quad \forall x, y \in R^{n},
$$

where $H(\cdot, \cdot)$ is the Hausdorff metric on closed bounded subsets of $R^{n}$ defined by

$$
H(A, B)=\max \left\{\sup _{u \in A} \operatorname{dist}(u, B), \sup _{v \in B} \operatorname{dist}(v, A)\right\} .
$$

The convergence rate of projection methods for GVI has been considered by many researchers $[20,22]$, and the following assumption is needed.

Assumption 16. Assume there are two positive constants $\mu$ and $\delta$ satisfying

$$
d\left(x, X^{*}\right) \leq \mu\|r(x, \xi)\|, \quad \forall x \text { with }\|r(x, \xi)\| \leq \delta,
$$

where $\xi \in F(x)$ and $d\left(x, X^{*}\right)$ denotes $l_{2}$-norm distance from $x$ to $X^{*}$.

Theorem 17. Let Assumptions 7 and 16 hold, and suppose the multivalued mapping $F$ is Lipschitz continuous with constant $L$. For the generated sequence $\left\{x^{k}\right\}$, the following statements hold:

(i) there is a constant $\tau>0$ such that, for all sufficiently large $k$,

$$
d\left(x, X^{*}\right) \leq \frac{\tau}{\sqrt{k+1}}
$$

(ii) as $X=R^{n}$, then

$$
\left\|x^{k+1}-x^{*}\right\|^{2} \leq\left[1-\frac{\sigma^{2}}{L^{2} \mu^{2}(1+\mu)^{2}}\right]\left\|x^{k}-x^{*}\right\|^{2},
$$

which means the sequence $\left\{x^{k}\right\}$ converges $R$-linearly to a solution of GVI.

Proof. (i) By the proof of Theorem 14, we know that there exists a positive constant $M$ such that

$$
\left\|\zeta^{k}\right\|^{2} \leq M, \quad \forall k
$$

From the fact that $\eta_{k} \leq 1$ for all $k$ and the proof of Theorem 14, we have

$$
\begin{aligned}
\left\|x^{k+1}-x^{*}\right\|^{2} & \leq\left\|x^{k}-x^{*}\right\|^{2}-\left(\alpha_{k}^{1}\right)^{2}\left\|\zeta^{k}\right\|^{2} \\
& =\left\|x^{k}-x^{*}\right\|^{2}-\frac{\left\langle\zeta^{k}, x^{k}-y^{k}\right\rangle^{2}}{\left\|\zeta^{k}\right\|^{2}} \\
& \leq\left\|x^{k}-x^{*}\right\|^{2}-\frac{\sigma^{2}}{M}\left\|r\left(x^{k}, \xi^{k}\right)\right\|^{4} .
\end{aligned}
$$

Since

$$
\left\|r\left(x^{k}, \xi^{k}\right)\right\| \longrightarrow 0, \quad \text { as } k \longrightarrow \infty
$$

one has

$$
\left\|r\left(x^{k}, \xi^{k}\right)\right\| \leq \delta, \quad \forall k \text { sufficiently large. }
$$

Choosing $x^{*} \in X^{*}$ such that

$$
d\left(x^{k}, X^{*}\right)=\left\|x^{k}-x^{*}\right\|
$$

we obtain

$$
\begin{aligned}
d\left(x^{k+1}, X^{*}\right) \leq & \left\|x^{k+1}-x^{*}\right\|^{2} \leq\left\|x^{k}-x^{*}\right\|^{2} \\
& -\frac{\sigma^{2}}{M}\left\|r\left(x^{k}, \xi^{k}\right)\right\|^{4} \leq\left\|x^{k}-x^{*}\right\|^{2} \\
& -\frac{\sigma^{2}}{\mu^{4} M}\left\|x^{k}-x^{*}\right\|^{4}=d\left(x^{k}, X^{*}\right)^{2} \\
& -\frac{\sigma^{2}}{\mu^{4} M} d\left(x^{k}, X^{*}\right)^{4},
\end{aligned}
$$

where the third inequality follows from Assumption 16. By Lemma 6 in Chapter 2 of [16], there exists a positive constant $\tau$ such that

$$
d\left(x, X^{*}\right) \leq \frac{\tau}{\sqrt{k+1}}
$$

for all $k$ sufficiently large.

(ii) If $X=R^{n}$, then the problem GVI reduces to the situation such that

$$
\exists x \in R^{n}, \quad 0 \in F(x) .
$$


Choosing $x^{*} \in X^{*}$ such that

$$
d\left(x^{k}, X^{*}\right)=\left\|x^{k}-x^{*}\right\|
$$

and by the fact that $F$ is Lipschitz continuous, we know that

$$
\begin{aligned}
\left\|\zeta^{k}\right\| & =\left\|\zeta^{k}-0\right\| \leq L\left\|y^{k}-x^{*}\right\| \\
& =L\left\|\left(1-\eta_{k}\right) x^{k}+\eta_{k} z^{k}-x^{*}\right\| \\
& =L\left\|\left(x^{k}-x^{*}\right)-\eta_{k} r\left(x^{k}, \xi^{k}\right)\right\| \\
& \leq L(1+\mu)\left\|r\left(x^{k}, \xi^{k}\right)\right\| .
\end{aligned}
$$

Hence,

$$
\begin{aligned}
\left\|x^{k+1}-x^{*}\right\|^{2} \leq & \left\|x^{k}-x^{*}\right\|^{2}-\left(\alpha_{k}^{1}\right)^{2}\left\|\zeta^{k}\right\|^{2}=\left\|x^{k}-x^{*}\right\|^{2} \\
& -\frac{\left\langle\zeta^{k}, x^{k}-y^{k}\right\rangle^{2}}{\left\|\zeta^{k}\right\|^{2}} \leq\left\|x^{k}-x^{*}\right\|^{2} \\
& -\frac{\sigma^{2}}{L^{2}(1+\mu)^{2}}\left\|r\left(x^{k}, \xi^{k}\right)\right\|^{2} \leq\left\|x^{k}-x^{*}\right\|^{2} \\
& -\frac{\sigma^{2}}{L^{2} \mu^{2}(1+\mu)^{2}}\left\|x^{k}-x^{*}\right\|^{2} \\
= & {\left[1-\frac{\sigma^{2}}{L^{2} \mu^{2}(1+\mu)^{2}}\right]\left\|x^{k}-x^{*}\right\|^{2}, }
\end{aligned}
$$

and it is obvious that $\left\{x^{k}\right\}$ converges $R$-linearly to $x^{*}$.

\section{Discussion}

Certainly, the proposed extragradient method for GVI in this paper has a good theoretical property in theory, as the generated sequence not only requires two projections at each iteration but also take different stepsize rules. Moreover, from a geometric point of view, it is shown that the new method has a long stepsize, and it guarantees that the distance from the next iterative point to the solution set has a large decrease. However, the proposed algorithm is not easy to be realized in practice as the residue and the trial point are not easy to execute. This is an interesting topic for further research.

\section{Acknowledgments}

This work was supported by the Natural Science Foundation of China (11171180) and the Specialized Research Fund for the Doctoral Program of Higher Education of China (20113705110002).

\section{References}

[1] A. Auslender and M. Teboulle, "Lagrangian duality and related multiplier methods for variational inequality problems," SIAM Journal on Optimization, vol. 10, no. 4, pp. 1097-1115, 2000.
[2] Y. Censor, A. Gibali, and S. Reich, "The subgradient extragradient method for solving variational inequalities in Hilbert space," Journal of Optimization Theory and Applications, vol. 148, no. 2, pp. 318-335, 2011.

[3] P. T. Harker and J.-S. Pang, "Finite-dimensional variational inequality and nonlinear complementarity problems: a survey of theory, algorithms and applications," Mathematical Programming, vol. 48, no. 2, pp. 161-220, 1990.

[4] Y. J. Wang, N. H. Xiu, and J. Z. Zhang, "Modified extragradient method for variational inequalities and verification of solution existence," Journal of Optimization Theory and Applications, vol. 119, no. 1, pp. 167-183, 2003.

[5] A. Ben-Tal and A. Nemirovski, "Robust convex optimization," Mathematics of Operations Research, vol. 23, no. 4, pp. 769-805, 1998.

[6] F. Facchinei and J. S. Pang, Finite Dimensional Variational Inequalities and Complementarity Problems, Springer, New York, NY, USA, 2003.

[7] C. Fang and Y. He, "A double projection algorithm for multivalued variational inequalities and a unified framework of the method," Applied Mathematics and Computation, vol. 217, no. 23, pp. 9543-9551, 2011.

[8] Y. He, "Stable pseudomonotone variational inequality in reflexive Banach spaces," Journal of Mathematical Analysis and Applications, vol. 330, no. 1, pp. 352-363, 2007.

[9] N.-J. Huang, "Generalized nonlinear variational inclusions with noncompact valued mappings," Applied Mathematics Letters, vol. 9, no. 3, pp. 25-29, 1996.

[10] S. Li and G. Chen, "On relations between multiclass, multicriteria traffic network equilibrium models and vector variational inequalities," Journal of Systems Science and Systems Engineering, vol. 15, no. 3, pp. 284-297, 2006.

[11] R. Saigal, "Extension of the generalized complementarity problem," Mathematics of Operations Research, vol. 1, no. 3, pp. 260266, 1976.

[12] Y. He, "The Tikhonov regularization method for set-valued variational inequalities," Abstract and Applied Analysis, vol. 2012, Article ID 172061, 10 pages, 2012.

[13] G. M. Korpelevich, "An extragradient method for finding saddle points and for other problems," Matecon, vol. 12, no. 4, pp. 747756, 1976.

[14] E. Allevi, A. Gnudi, and I. V. Konnov, "The proximal point method for nonmonotone variational inequalities," Mathematical Methods of Operations Research, vol. 63, no. 3, pp. 553-565, 2006.

[15] Y. J. Wang, N. H. Xiu, and C. Y. Wang, "Unified framework of extragradient-type methods for pseudomonotone variational inequalities," Journal of Optimization Theory and Applications, vol. 111, no. 3, pp. 641-656, 2001.

[16] B. T. Polyak, Introduction to Optimization, Optimization Software Incorporation, Publications Division, New York, NY, USA, 1987.

[17] D. Kinderlehrer and G. Stampacchia, An Introduction to Variational Inequalities and Their Applications, Academic Press, New York, NY, USA, 1980.

[18] J.-P. Aubin and I. Ekeland, Applied Nonlinear Analysis, John Wiley \& Sons, New York, NY, USA, 1984.

[19] Y. He, "A new double projection algorithm for variational inequalities," Journal of Computational and Applied Mathematics, vol. 185, no. 1, pp. 166-173, 2006. 
[20] M. V. Solodov, "Convergence rate analysis of iteractive algorithms for solving variational inquality problems," Mathematical Programming, vol. 96, no. 3, pp. 513-528, 2003.

[21] J. S. Pang, "Error bounds in mathematical programming," Mathematical Programming, vol. 79, no. 1-3, pp. 299-332, 1997.

[22] F.-Q. Xia and N.-J. Huang, "A projection-proximal point algorithm for solving generalized variational inequalities," Journal of Optimization Theory and Applications, vol. 150, no. 1, pp. 98-117, 2011. 


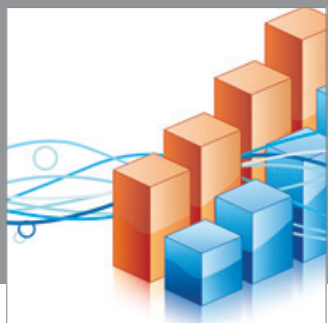

Advances in

Operations Research

mansans

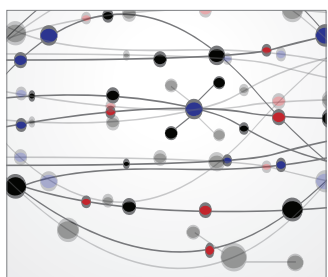

The Scientific World Journal
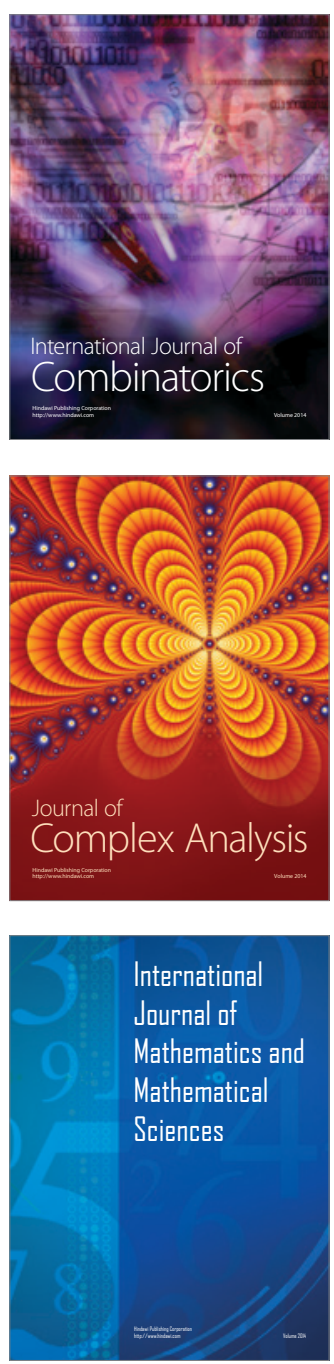
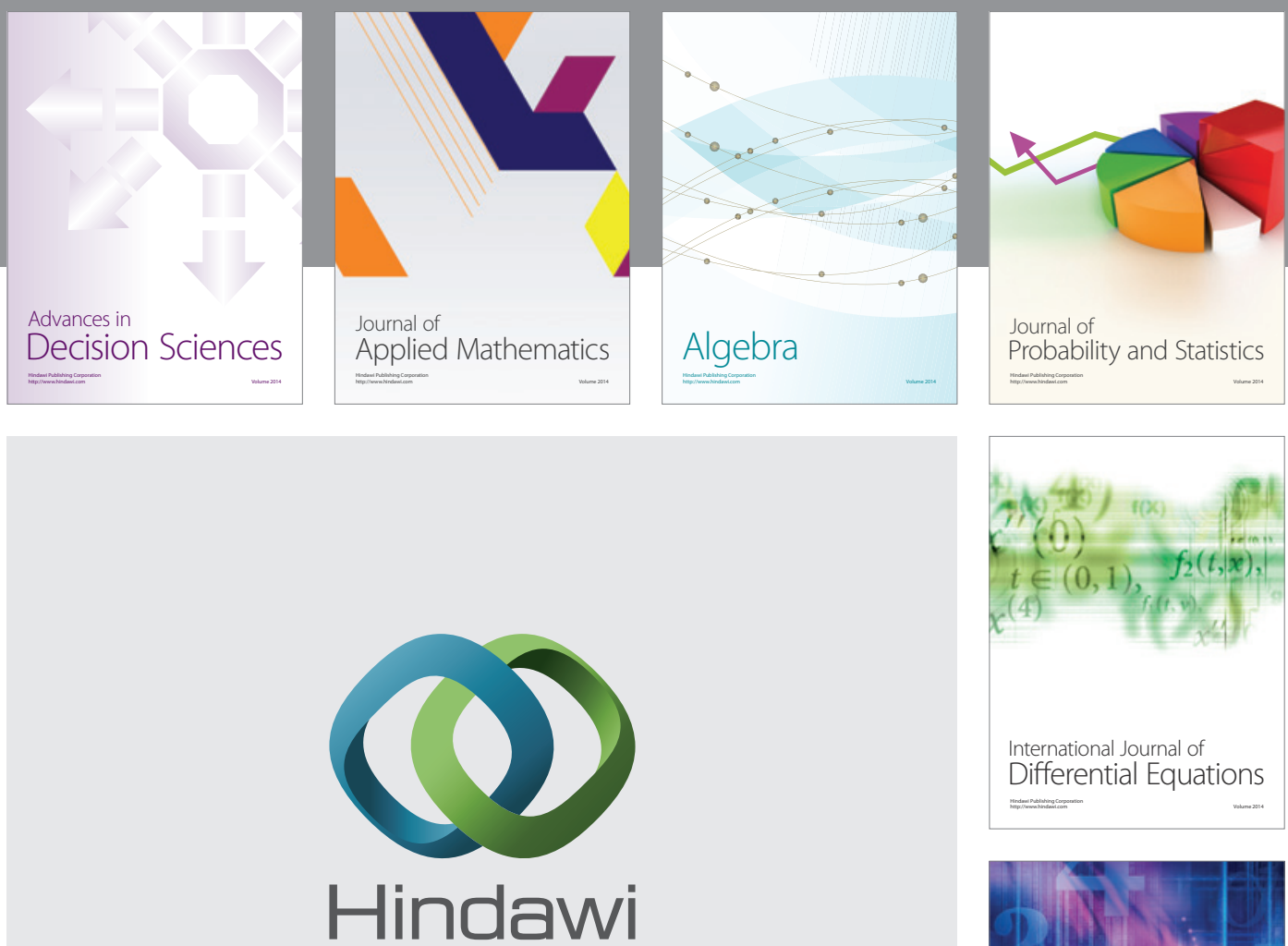

Submit your manuscripts at http://www.hindawi.com
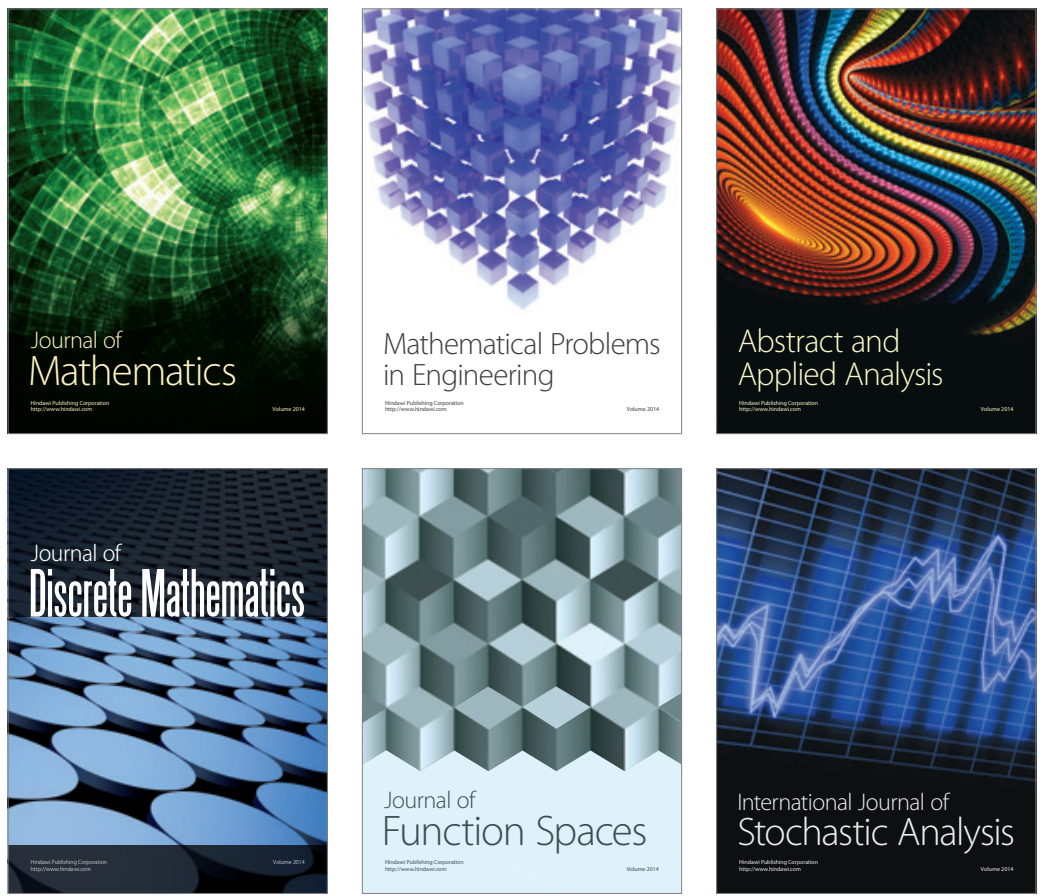

Journal of

Function Spaces

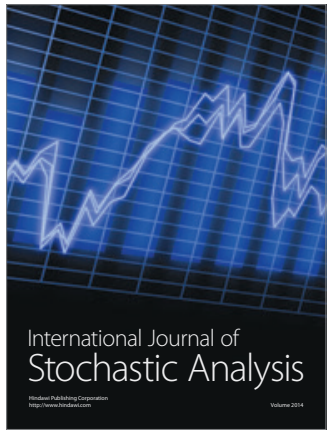

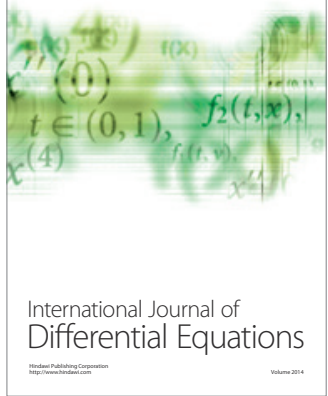
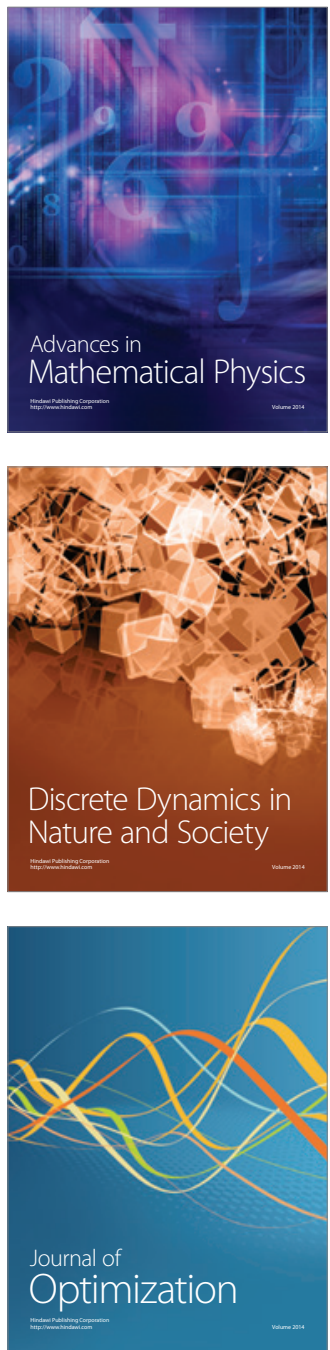